\title{
Accretion bursts in young stars driven by cluster environment
}

\author{
S. Pfalzner, J. Tackenberg, M. Steinhausen
}

\author{
I. Physikalisches Institut, University of Cologne, Zülpicher Str. 77, 50937 Cologne, Germany
}

\begin{abstract}
Context. The standard picture of accretion is a steady flow of matter from the disc onto the young star - a concept which assumes the star-disc system to be completely isolated. However, in a dense cluster environment star-disc systems do interact gravitationally.

Aims. The aim here is to estimate the encounter-induced accretion rate in an ONC-like environment.

Methods. Combining simulations of the cluster dynamics with simulations of the effect of encounters on star-disc systems we determine the likelihood and degree of encounter-triggered accretion processes.

Results. We show that accretion bursts triggered by encounters of star-disc systems are common in young dense clusters like the ONC leading in the outburst phase to typical accretion rates of $10^{-7}-10^{-4} \mathrm{M}_{\odot} / \mathrm{yr}$. Up to a third of stars presently in the Trapezium region accreted at least $1 \%$ of their disc mass via this mechanism in the last $1 \mathrm{Myr}$. Accretion of over 6-7\% of the disc material can occur in a single encounter. Despite losing their discs quickly, the total percentage of disc matter accreted per star is largest for the massive stars.

Conclusions. Supplementing the steady accretion flow there exist episodic periods of high accretion in dense cluster environments. Due to their high accretion rate these processes should be observable even now in some of the low-mass stars in the ONC.
\end{abstract}

Key words. young clusters - protoplanetary discs - circumstellar matter - ONC

\section{Introduction}

The theory of accretion has been extensively studied in the context of the standard accretion disc model (Pringle \& Rees 1972; Shakura 1973; Pringle 1981). The main idea is that of gas moving inward due to radial turbulent transport of angular momentum to the outer disc regions. Currently the magneto-rotational instability (Balbus \& Hawley 1991) seems to be able to provide the required degree of turbulence in accretion discs.

The disc evolution that follows is not completely understood. In the later phases accretion happens exclusively from the disc material, so linking disc development and accretion directly. The often-used theoretical models of viscous disc evolution (Lynden-Bell \& Pringle 1974; Hartmann et al. 1998) treat the disc as an isolated smooth axisymmetric structure that evolves due to an unspecified source of temporally independent, turbulent viscosity. These standard models assume a possibly decreasing with time but relatively steady accretion process. Recently models were suggested where accretion is caused by gravitational instabilities in the disc (Vorobyov \& Basu 2006; Boley et al. 2006) leading to alternating phases of high and low accretion. However, for these instabilities to occur the disc has to be relatively massive $\left(m_{d}>0.1 M^{*}\right)$, which means it will predominantly occur very early in star formation.

From observations the general picture emerges that the disc fraction is a strong function of age, decreasing from $\sim 80 \%$ for clusters at 1 Myr down to few discs for clusters older than 10 Myr (Haisch et al 2001; Hernandez 2007). The typical accretion rate for a young star is $\sim 10^{-8}-10^{-10} \mathrm{M}_{\odot} / \mathrm{yr}$ (Hillenbrand et al. 1995;
Gullbring 1998; Haisch et al 2001). However, the development of the disc and the accretion rate of individual sources in the same environment can differ considerably (Flaherty \& Muzerolle 2008). The accretion rate depends on the stellar age, mass and environment. As expected from viscous disc models a decrease in accretion rates with stellar age has been found (Hartmann et al. 1998; Muzerolle et al. 2000). A matter of debate is the dependence on the stellar mass of the form $M^{\alpha}$ (Calvet et al. 2004; Sicilia-Aquilar et al. 2006; Natta et al. 2006), possibly with $\alpha \sim 2$. The effect of the environment possibly manifests itself in that Herbig Ae stars have on average higher accretions rates (Lopes et al. 2006) than low-mass stars in Taurus and Ophiuchus.

Here we investigate an accretion process that will be present in dense cluster environments in addition to the steady accretion - the passage of a star inducing a simultaneous transport of disc matter outwards and inwards via spiral arms, leading to accretion. We will show that the consequences are short bursts of high accretion $\left(10^{-7}-10^{-4}\right.$ $\left.\mathrm{M}_{\odot} / \mathrm{yr}\right)$, inevitably occuring in dense clusters and observable due to these high accretion rates.

Although this process has properties in common with accretion induced by gravitational instabilities (Vorobyov \& Basu 2006; Bolev et al. 2006) and in wide binaries (Bonnell \& Bastien 1992), the cluster dependence is unique to the process treated here.

For encounters to play an important role the cluster has to be dense. A typical example for such an environment is the Orion Nebula Cluster(ONC). In an earlier paper (Pfalzner 2006), we demonstrated that encounters cause a $3-5 \%$ specific angular momentum loss in the ONC rising to $10-15 \%$ in the dense inner Trapezium region. Since specific 
angular momentum loss is a prerequisite for accretion we suggested that in the final star formation stages an additional growth mechanism for massive stars exist (clusterassisted accretion) as massive stars lose their specific angular momentum to a higher degree than low-mass stars. Here we study encounter-induced accretion directly by measuring the amount of matter kicked into the inner disc areas. Obviously this is not strictly accretion, but represents a often-used method in circumstances where computational expense does not allow to further resolve the inner disc (Bate et al. 2002; Vorobyov \& Basu 2005). In the following the term "accreted" will be used in this sense but the limitations of this approach should be kept in mind.

\section{Method}

We combine simulations of the cluster dynamics performed with the Nbody6++ code (Spurzem 1999) with tree code simulations to study the effect of encounters on star-disc systems as described in Pfalzner (2006). Here we determine the likelihood and degree of encounter-triggered accretion processes. For simplicity all stars of the cluster are assumed to be initially single, the effect of gas and the potential of the background molecular cloud OMC 1 are neglected. Cluster models were set up with a spherical density distribution $\rho(r) \propto r^{-2}$ and a Maxwell-Boltzmann velocity distribution. $\theta^{1} \mathrm{C}$ Ori was placed at the cluster centre and assigned a mass of $50 \mathrm{M}_{\odot}$. For all other stars the masses were generated randomly according to the mass function given by (Kroupa 2002) in a range $50 \mathrm{M}_{\odot} \geq M^{*} \geq 0.08 \mathrm{M}_{\odot}$. The Nbody6++ code determines the trajectories of the stars to a high precision (Aarseth 2003, see). Information of all perturbing events of each stellar disc was recorded during the simulation, i.e. both masses, the relative velocity and the eccentricity. More details about the encounter simulations can be found in the appendix. The observed peak density of the Trapezium region of $4.7 \times 10^{4} \mathrm{stars} / \mathrm{pc}^{-3}$ was reproduced in our simulations within $20 \%$, as was the stellar density distribution of the ONC. We chose the ONC to be in virial equilibrium.

The code applied here cannot describe the disc dynamics simultaneously with the accretion process. The mass density - as the required number of simulation particles would be too high close to the star and so would be the required temporal resolution. In any case the real accretion process will be rather complex. The main question is whether perturbations to the inner disc area can be induced by an encounter. Whether this is done directly by a particle stream or only some related mechanism is of secondary importance. Therefore we resort to the approach of Bate et al (2002) and Vorobyov \& Basu (2005), by computing the amount of matter reaching a sphere around the central star (here 1AU). We model only gravitational interactions, hydrodynamical effects have been neglected. In principle the latter could hinder matter reaching the inner disc areas due to pressure gradient. However, here the velocity of the instreaming matter is so high that this is in most cases not a problem. Nevertheless the accretion values attributed here should only be regarded as relative values, determining actual accretion rates would require a more sophisticated approach. The present study only aims at determining in which context encounters are likely to trigger accretion processes.

\section{Encounter results}

First we determine how much disc mass is accreted depending on the encounter parameters. In principle, the star can not only accrete matter from its own disc but from the disc of the passing star as well. We find this only happens in very close or penetrating encounters and so this process is not included in the following. We performed a parameter study of coplanar, parabolic encounters, which shows that in a single fly-by up to $6-7 \%$ of the disc mass (Fig. A.1 and Table A.1) is accreted. In general much more mass is lost than accreted, with typical ratios of $m_{\text {accreted }} / m_{\text {lost }} \sim 2-5$ $\%$, which can rise to $\sim 10 \%$ in some cases. Even fly-bys as distant as $10 r_{d}$, where $r_{d}$ is the disc size $(150 \mathrm{AU})$, can induce accretion provided that the mass ratio $M_{2}^{*} / M_{1}^{*}$ of the two interacting stars is high enough, where $M_{1}^{*}$ indicates the mass of the considered star and $M_{2}^{*}$ the mass of the passing star. For $M_{2}^{*} / M_{1}^{*} \leq 1$ the amount of accreted matter simply increases the closer the encounter, but for very small mass ratios, $M_{2}^{*} / M_{1}^{*}<0.1$, the accreted matter never exceeds $1 \%$ of the disc mass regardless of how close the star passes. For $M_{2}^{*} / M_{1}^{*}>1$ the higher mass passing star increasingly captures matter that otherwise would have been accreted by the primary. Note, although the above results were obtained for discs with masses $m_{d}=0.001 \mathrm{M}_{\odot}$, they are applicable for $\sim m_{d}>0.5 M_{1}^{*}$. For a discussion see Appendix.

Typical disc masses are $m_{d} \sim 0.01 M \odot$ and accretion bursts last normally $\sim 10^{2}-10^{3}$ yrs. So $5 \%$ accreted disc mass is roughly equivalent to an averaged accretion rate of $5 \times 10^{-7}-10^{-6} \mathrm{M}_{\odot} / \mathrm{yr}$. However, there will be a wide range of induced accretion rates, as the disc mass depends strongly on the stellar age and mass.

So far we considered only prograde encounters, but what happens in retrograde encounters? As long as the passing star does not penetrate the disc, no matter becomes accreted. However, encounters closer than $0.5 r_{d}$ can lead to higher accretion rates in retrograde than in prograde encounters. As distant encounters are more common using the accretion rates from prograde encounters leads probably to a somewhat overestimated accretion.

Cluster dynamics simulations show that especially in the dense inner regions stars often undergo a series of encounters (Olczak et al. 2006). We simulated consecutive encounters with the same parameters but using the resulting density distribution as input for the next encounter. Each encounter reduces the disc mass and alters the density distribution. The result is that in subsequent encounters less matter is accreted. To approximate this, we use the results from single encounters but relative to the reduced disc mass determined using Eq. (4) from Olczak et al. (2006).

In the following we combine above encounter results with cluster simulations described in (Olczak et al. 2006).

\section{Cluster-induced accretion}

For ONC conditions nearly all cluster-induced accretion processes happen within $0.2 \mathrm{pc}$ around the cluster center. Here the high stellar density leads to a large number of encounters $(>500$ within 0.03 pc compared to $<50$ outside $0.2 \mathrm{pc})$.

After $\sim 0.1$ Myr about a third of all stars presently in the dense Trapezium region have accreted at least $1 \%$ of their disc mass (see Fig. (1). In other words, these stars 


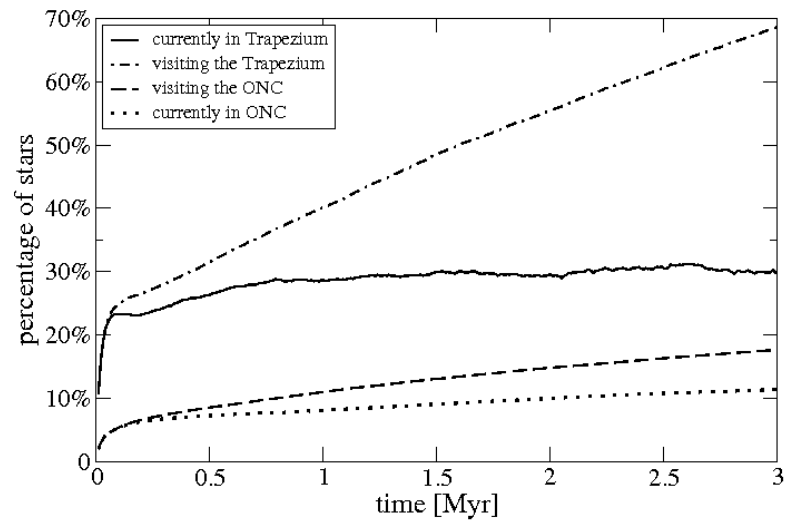

Fig. 1. Percentage of stars currently in Trapezium and ONC region with $<1 \%$ of the disc mass accreted as function of time and the same for stars that transversed these areas in their past.

a)
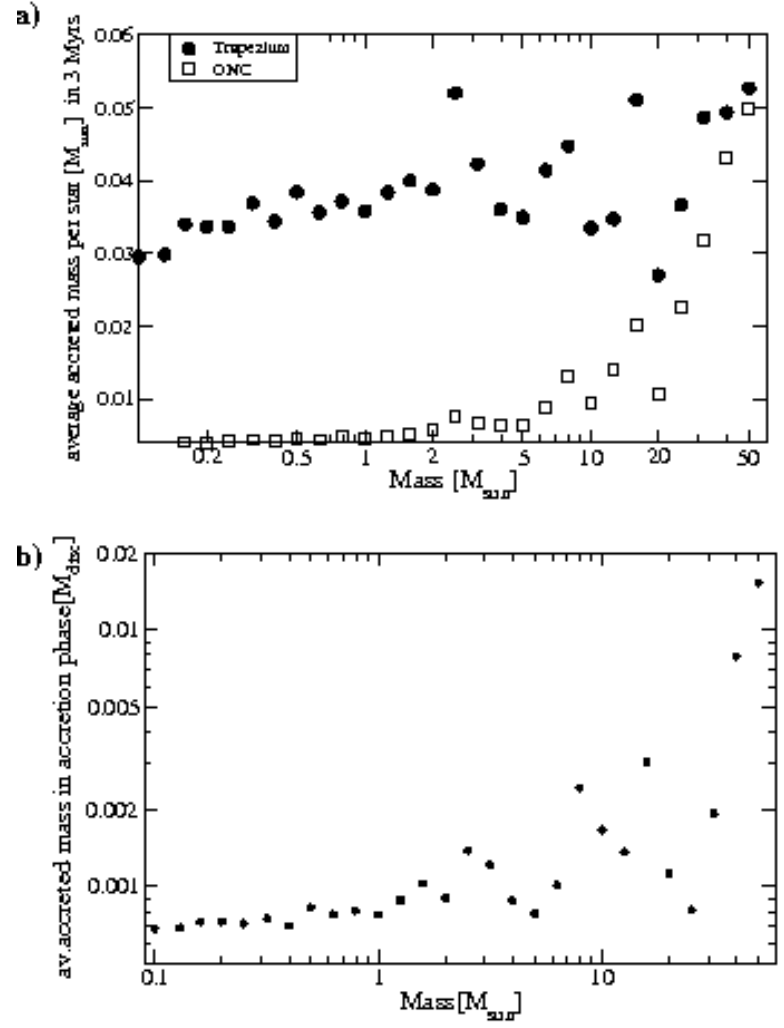

Fig. 2. a) Mass accreted per encounter in the Trapezium region and the entire ONC averaged over the first 3 Myrs and b) mass accreted per star during the accretion phases shown as a function of primary mass in units of disc masses.

have shown signs of very high accretion $\left(>10^{-7} \mathrm{M}_{\odot} / \mathrm{yr}\right)$ due to interacting with neighbouring stars in the past 1-2 Myr. The stellar content fluctuates, so looking at stars that have visited the Trapezium region in the past, this fraction will increases to $\sim 70 \%$ by $3 \mathrm{Myr}$.

Fig. 2a) shows the accreted matter per star as a function of the primary mass $M_{1}^{*}$ during the first $3 \mathrm{Myr}$. In the Trapezium region the accreted matter rises by a factor of $\sim 1.5$ from low-mass to massive stars. In the entire ONC the dependency is much stronger as many more low-mass stars never undergo an encounter leading to accretion. Here the average accreted mass per star is 10-15 times larger for massive than for low-mass stars.

We find that the accreted mass per accreting encounter is nearly constant up to $M_{1}^{*} \sim 5 \mathrm{M}_{\odot}$ and rises for higher masses. The average number of accreting encounters is also higher for massive stars. In the Trapezium region a $20 \mathrm{M}_{\odot}$ star has on average more than twice the number of accreting encounters than a $0.1 \mathrm{M}_{\odot}$ star; this factor increases to 10 times when considering the entire ONC. However, the most massive stars lose their discs quickly (typically $\sim 5 \times 10^{5}$ yrs) so that although they undergo encounters there is no matter left to be accreted.

Nevertheless, the accreted mass is highest for the most massive stars. The mass dependence can be most clearly seen looking at the average accreted mass in the accretion phase - meaning the time until the star has completely lost its disc. Fig. 20) shows that the accreted mass is more than 30 times more for massive stars than low-mass stars.

The number of accreting encounters decreases strongly with cluster age, so are there currently any observable encounter-triggered accretion bursts in the ONC happening? Although each individual low-mass star is less likely to undergo an accreting encounter than massive stars due to their relative abundance, one is more likely to observe a low-mass star having such an encounter than stars with higher mass (see Fig. 3). For the low-mass stars the number of encounters decreases from several hundred in the first $10^{4}$ yrs to 5 - 10 such events in an timeinterval of $10^{4}$ yrs at the current age of $\sim 1 \mathrm{Myr}$. As most encounter triggered accretion processes last $10^{3}-10^{4}$ yrs, this means that one can expect to find currently between 1 and 10 low-mass stars in the ONC that show high accretion rates due to encounter processes.

\section{Discussion and Conclusion}

There is one observed systems that displays the link between encounter and accretion rate. The morphology and kinematics (Cabrit et al. 2006) of RW Aurigae A and B strongly suggest tidal stripping of the primary disc by a recent fly-by that is occurring. This system displays a high accretion rate of $\sim 2-10 \times 10^{-7} \mathrm{M}_{\odot} / \mathrm{yr}$ (Basri \& Bertout 1989; Hartigan et al. 1995) while having a particularly low disc mass of $m_{d}^{c} \sim 3 \times 10^{-4} \mathrm{M}_{\odot}$. Using these parameters our simulations show the likely total accreted mass to be $0.3-4 \%$ of the pre-encounter mass, equivalent to a maximum induced accretion rate of $1 \cdot 10^{-6}$ - $1.2 \cdot 10^{-5} \mathrm{M}_{\odot} / \mathrm{yr}$ assuming a pre-encounter disc mass $\left.m_{d}^{\text {pre }}<10 \times m_{d}^{c}\right)$ and that most mass is accreted within $\sim 10$ years (although high accretion for several 100 years exist). This accretion rate is consistent with the observed accretion rate of $\sim 2-10 \cdot 10^{-7} \mathrm{M}_{\odot} / \mathrm{yr}$. So encounterinduced accretion is probably occuring in this system.

However, RW Aurigae is not located in a dense cluster, so either the rare case of an encounter in a low-density region or a strongly elliptical wide binary is being observed. Even in the latter case, the results described in Section 3 are still applicable as the effect of a parabolic encounter on the induced accretion is very similar to that in a strongly elliptical wide binary. Episodes of rapid accretion due to tidal disturbances to the disc induced by a binary companion were suggested by Bonnell and Bastien (1992) in the context of FU Orionis flareups, which are 


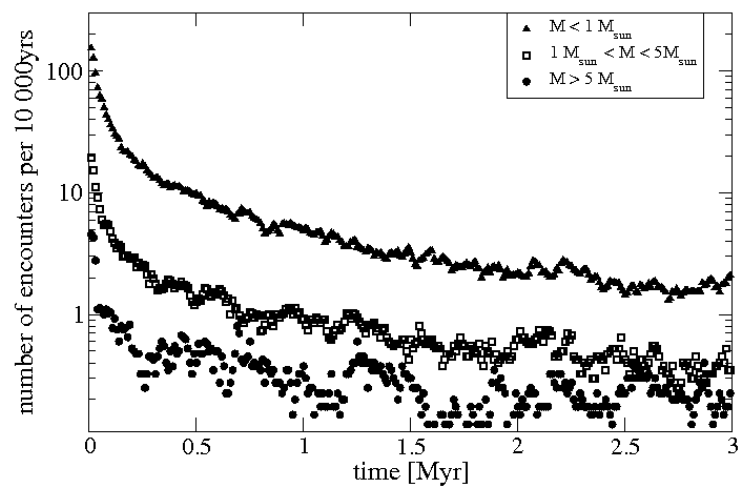

Fig. 3. Average number of encounters in any time interval of 10000 yrs that lead to accretion as a function of cluster age.

believed to be powered by episodes of exceptionally rapid accretion (up to $10^{-4} \mathrm{M}_{\odot} / \mathrm{yr}$ ) (Hartmann \& Kenyon 1996). Alternatively, such outbursts could be due to thermal instability (Bell \& Lin 1994; Bell 1999), or to gravitational instability in the disc (Vorobyov \& Basu 2006). While many of the properties (accretion rate, duration of burst) of the accretion bursts described here agree with those of FUors, the strongest argument against it is the lack of FUors observed in dense stellar regions. The arguments for and against a connection of encounter-triggered accretion bursts to FU Orionis objects will be discussed in more detail in Pfalzner (2008).

Accretion bursts might be even more important in the earlier phases where star-disc systems are still in their formation process (Larson 2002). The simulation of the formation of a small cluster of stars by Bate (2002) clearly shows the formation of many circumstellar discs. The complex dynamics of the system includes frequent protostellar interactions disrupting the discs by encounters. However new discs soon form from gas that continues to fall inwards, so that in this phase protostars may gain much of their mass in discrete episodes of encounter-triggered accretion.

The observed accretion rates in Class I protostars are orders of magnitude smaller than those needed to form a star during the lifetime of a Class I object (McKee \& Ostriker 2007). Kenyon et al. (1990) suggested two solutions to this problem - either significant accretion continues into the $\mathrm{T}$ Tauri stage or most of the accretion occurs in the embedded stage. The former appears to be ruled out because such stars accrete very slowly with no significant disc or envelope mass reservoir that they can draw on. In the latter case accretion could be episodic, so that the median accretion rate is much smaller than the mean.

Another fact that can be explained if the accretion occurs in short bursts is that the observed luminosities of many protostars are lower than is predicted for models with steady accretion (Kenyon et al. 1995; Calvet et al. 2004). The jet-like Herbig-Haro outflows probably powered by rapid accretion onto forming stars at early stages of evolution, are episodic or pulsed, suggesting that the accretion process is itself episodic (Reipurth 2001). Remarkably the jet sources are frequently (in $85 \%$ of cases) found to have close stellar companions (Larson 2002).

\section{Summary}

We have demonstrated that encounter-induced bursts of accretion are common phenomena in the centre of young dense clusters. Successive encounters can lead to over $10 \%$ of the disc mass being accreted typically with accretion rates $>10^{-7} \mathrm{M}_{\odot} / \mathrm{yr}$. In young dense cluster environments these episodal accretion processes will inevitably happen in addition to the steady viscous accretion of disc material. We showed that accretion-inducing encounters are more likely and lead to higher accretion rates for massive stars.

\section{References}

Aarseth, S. J. 2003, Gravitational N-Body Simulations, CUP, Cambridge, UK.

Alexander, R.D. \& Armitage,P.J. 2006, ApJ, 693, L83.

Balbus, S.A. and Hawley, J.F. 1991, ApJ, 376, 214.

Basri, G. and Bertout, C. 1989, ApJ, 341, 340.

Bate, M. R. Bonnell, I. A., Bromm, V. 2002 MNRAS336, 705.

Bell, K. R., Lin, D. N. C. 1996, ApJ, 427, 987.

Bell, K. R. 1999, ApJ, 526, 411.

Boley, A. C., Mejia, A. C., Durisen, R.H. Cai, K., Pickett, M. K., D'Alessio, P. 2006, ApJ, 651, 517

Bonnell, I. \& Bastien, P. 1992, ApJ, 401, L31

Cabrit, S., Pety, J., Pesenti, N., Dougados, C. 2006, A\&A, 452, 897.

Calvet, N., Muzerolle, J., Briceno, C., Hernandez, J., Hartmann, L., Saucedo, J., Gordon, K. 2004, AJ, 128, 1294.

Clarke, C. \& Pringle, J.E. 2006, MNRAS, 370, L10.

Dullemond, C. P., Natta, A., Testi, L. 2006, ApJ, 645, L69.

Flaherty, K.M. \& Muzerolle, J. 2008, astro-ph 07121601

Gullbring, E. Hartmann, L., Briceno, C., Calvet, N. 1998 ApJ 492, 323.

Haisch, K., Lada, E. A., Lada, C. J. 2001, ApJ, 553, 1153.

Hartigan, P., Edwards, S., Ghandour, L. 1995, ApJ, 452, 736.

Hartmann, L., 1998, ApJ, 495, 385.

Hartmann, L., D'Allessio, Calvet, Muzerolle 2006, ApJ, 648, 484.

Hartmann, L. \& Kenyon, S. J. 1996, ARA\&A, 34, 207

Hernandez, J., Calvet, N., Briceno, C., Hartmann, L., Vivas, A. K., Muzerolle, J., Downes, J., Allen, L., Gutermuth, R. 2007, ApJ 671, 1784 .

Hillenbrand, L. A. et al. 1995, AJ, 116, 1816.

Kenyon, S. J., Hartmann, L. W., Strom, K. M., Strom, S. E.1990, AJ 99, 869.

Kenyon, S. J. \& Hartmann, L. 1995 ApJS 101, 117.

Kroupa, P. 2002, 295, 82.

Larson, R. B. 2002 MNRAS, 332, 155.

Lopes, R.G., Natta, A., Testi, L., Habart, E. 2006, astro-ph/0609032 Lynden-Bell, D. \& Pringle, J. E. 1974, MNRAS, 168, 603.

McCaughrean, M., Zinnecker, H., Andersen, M., Meeus, G., Lodieu, N. 2002, The Messenger, 109, 28.

McKee, C. F. \& Ostriker, E. C. 2007, ARAA 45, 565.

Muzerolle, J., Calvet, N., Briceno, C., Hartmann, L., Hillenbrand, L. 2000, ApJ, 535, L47.

Muzerolle, J., Luhmann, K.L., Briceno, C., Hartmann, L., Calvet, N. 2005, ApJ, 617, 406.

Natta, A. Testi,L., Muzerolle, J., Randich. S., Comeron, F., Persi, P. 2004, A\&A, 424, 603.

Natta, A., Testi, L., Randich, S. 2006, A\&A, 452, 245.

Olczak, C., Pfalzner, S., Spurzem, R. 2006, ApJ, 642, 1140.

Ostriker, E.C. 1994, ApJ, 424, 292.

Pfalzner, S. 2006, ApJ, 652, L129.

Pfalzner, S. 2008, in preparation.

Pfalzner, S., Olczak, C., Eckart, A. 2006, A\&A, 454, 811.

Pringle, J. E. 1981 ARAA, 19, 137.

Pringle, J. E. \& Rees, M. J. 1972, A\&A, 21, 1.

Reipurth, B., Bally, J. 2001 ARAA 39, 403.

Shakura, N. I. 1973, SA, 16, 756.

Sicilia-Aguilar, A., Hartmann, L., Frsz, G., Henning, Th., Dullemond, C., Brandner, W. 2006, AJ 132, 2135

Spurzem, R. 1999, Comp. Astroph., 109, 407. 
Vorobyov, E. I., Basu, S. 2005, ApJ633, L137

Vorobyov, E. I., Basu, S. 2006, ApJ650, 956 


\section{Appendix A: Parameter study}

\section{A.1. Encounter simulations}

In the encounter simulations themselves the mass of the discs was assumed to be $m_{d}=0.001 \mathrm{M}_{\odot}$ as is typical for $\mathrm{T}$ Tauri stars. However, the results are applicable for higher disc masses as well provided self-gravity effects do not play a dominant role. The main effect would be a rearranging of the disc material and a deviation from the Keplerian velocities of the disc particles. This probably happens for $m_{d} \sim M_{1}^{*}$. For such massive discs the cluster simulation particles would need to represent the mass of the star disc system and not just the stars as is currently the case. In this situation the encounter rate is likely to rise slightly increasing the number of encounter-induced accretion events even more. But this situation would need additional investigations.

In the encounter simulations the disc size was assumed to be 100 AU. But as the cluster consists of a wide spectrum of stellar masses, the simulation results, valid for $M_{1}^{*}=1 M_{\odot}$, are generalized by scaling the disc radius according to $r_{\mathrm{d}}=150 \sqrt{M_{1}^{*}\left[\mathrm{M}_{\odot}\right]}$, which is equivalent to the assumption of a fixed force at the disc boundary. The quality of the dynamical models were determined by comparing them to observational data (McCaughren et al. 2002) at the approximate age of the ONC (1-2Myr). The quantities of interest were: number of stars, half-mass radius, number densities, velocity dispersion and projected density profile. For more details of the selection process, see (Olczak et al. 2006).

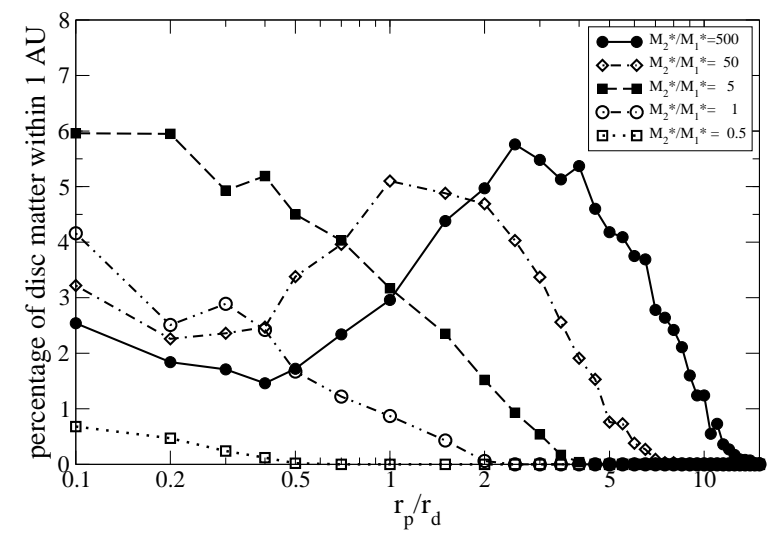

Fig. A.1. Percentage of disc mass accreted onto star as function of the ratio of periastron $r_{p}$ to the disc radius $r_{d}$ for different $M_{2}^{*} / M_{1}^{*}$ ratios in parabolic, prograde coplanar encounters. 
Pfalzner et al.: Accretion bursts in young stars, Online Material $p 2$

\begin{tabular}{|c|c|c|c|c|c|c|c|c|c|c|c|c|c|c|}
\hline & 500.0 & 90.0 & 50.0 & 20.0 & 9.0 & 5.0 & 4.0 & 3.0 & 2.0 & 1.5 & 1.0 & 0.5 & 0.3 & 0.1 \\
\hline 0.1 & 2.54 & 2.86 & 3.22 & 6.94 & 7.83 & 5.96 & 5.85 & 5.10 & 4.33 & 4.22 & 4.16 & 4.26 & 4.87 & 0.68 \\
\hline 0.2 & 1.84 & 1.67 & 2.26 & 4.05 & 5.30 & 5.95 & 6.22 & 5.33 & 5.98 & 4.97 & 2.51 & 3.44 & 2.61 & 0.47 \\
\hline 0.3 & 1.71 & 2.21 & 2.36 & 2.92 & 4.10 & 4.93 & 4.90 & 5.68 & 5.68 & 5.07 & 2.89 & 2.82 & 1.84 & 0.24 \\
\hline 0.4 & 1.46 & 2.53 & 2.47 & 3.24 & 4.50 & 5.19 & 4.78 & 4.28 & 4.01 & 3.51 & 2.42 & 2.15 & 1.26 & 0.12 \\
\hline 0.5 & 1.72 & 2.68 & 3.38 & 4.26 & 4.67 & 4.50 & 3.81 & 3.71 & 3.53 & 2.11 & 1.67 & 1.23 & 0.59 & 0.02 \\
\hline 0.7 & 2.34 & 3.65 & 3.96 & 4.77 & 4.91 & 4.03 & 3.74 & 3.14 & 2.24 & 1.83 & 1.22 & 0.54 & 0.22 & 0 \\
\hline 1.0 & 2.96 & 5.01 & 5.10 & 4.59 & 4.13 & 3.17 & 2.99 & 2.23 & 1.74 & 1.61 & 0.87 & 0.30 & 0.03 & 0 \\
\hline 1.5 & 4.38 & 4.51 & 4.88 & 4.57 & 2.93 & 2.35 & 1.85 & 1.46 & 1.03 & 0.87 & 0.43 & 0.01 & 0 & 0 \\
\hline 2.0 & 4.97 & 5.79 & 4.69 & 3.36 & 2.09 & 1.52 & 1.38 & 1.33 & 0.74 & 0.44 & 0.06 & 0 & 0 & 0 \\
\hline 2.5 & 5.76 & 4.90 & 4.03 & 2.40 & 1.69 & 0.93 & 0.79 & 0.43 & 0.17 & 0.07 & 0 & 0 & 0 & 0 \\
\hline 3.0 & 5.48 & 4.11 & 3.37 & 1.81 & 0.98 & 0.54 & 0.47 & 0.14 & 0.03 & 0.01 & 0 & 0 & 0 & 0 \\
\hline 3.5 & 5.13 & 3.60 & 2.56 & 1.76 & 0.63 & 0.17 & 0.04 & 0.07 & 0 & 0 & 0 & 0 & 0 & 0 \\
\hline 4.0 & 5.37 & 3.02 & 1.91 & 0.79 & 0.14 & 0.04 & 0.01 & 0 & 0 & 0 & 0 & 0 & 0 & 0.0 \\
\hline 4.5 & 4.60 & 2.31 & 1.53 & 0.56 & 0.11 & 0 & 0 & 0 & 0 & 0 & 0 & 0 & 0 & 0 \\
\hline 5.0 & 4.18 & 1.76 & 0.76 & 08 & 02 & 0 & 0 & 0 & 0 & 0 & 0 & 0 & 0 & 0 \\
\hline 5.5 & 4.09 & 1.56 & 0.73 & 0.11 & 0 & 0 & 0 & 0 & 0 & 0 & 0 & 0 & 0 & 0 \\
\hline 6.0 & 3.75 & 1.25 & 0.38 & 03 & 0 & 0 & 0 & 0 & 0 & 0 & 0 & 0 & 0 & 0 \\
\hline 6.5 & 3.69 & 0.68 & 0.27 & 0 & 0 & 0 & 0 & 0 & 0 & 0 & 0 & 0 & 0 & 0 \\
\hline 7.0 & 2.78 & 0.45 & 09 & 0 & 0 & 0 & 0 & 0 & 0 & 0 & 0 & 0 & 0 & 0 \\
\hline 7.5 & 2.64 & 0.15 & 03 & 0 & 0 & 0 & 0 & 0 & 0 & 0 & 0 & 0 & 0 & 0 \\
\hline 8.0 & 2.42 & 09 & 03 & 0 & 0 & 0 & 0 & 0 & 0 & 0 & 0 & 0 & 0 & 0 \\
\hline 8.5 & 2.11 & 03 & 0 & 0 & 0 & 0 & 0 & 0 & 0 & 0 & 0 & 0 & 0 & 0 \\
\hline 9.0 & 1.60 & 02 & 0 & 0 & 0 & 0 & 0 & 0 & 0 & 0 & 0 & 0 & 0 & 0 \\
\hline 9.5 & 1.24 & 01 & 0 & 0 & 0 & 0 & 0 & 0 & 0 & 0 & 0 & 0 & 0 & 0 \\
\hline 10. & 1.24 & 0 & 0 & 0 & 0 & 0 & 0 & 0 & 0 & 0 & 0 & 0 & 0 & 0 \\
\hline 10.5 & 0.55 & 0 & 0 & 0 & 0 & 0 & 0 & 0 & 0 & 0 & 0 & 0 & 0 & 0 \\
\hline 11.0 & 0.73 & 0 & 0 & 0. & 0 & 0 & 0 & 0 & 0 & 0 & 0 & 0 & 0 & 0 \\
\hline 11.5 & 0.36 & 0 & 0 & 0 & 0 & 0 & 0 & 0 & 0 & 0 & 0 & 0 & 0 & 0 \\
\hline 12.0 & 0.27 & 0 & 0 & 0 & 0 & 0 & 0 & 0 & 0 & 0 & 0 & 0 & 0 & 0 \\
\hline 12.5 & 0.17 & 0 & 0 & 0 & 0 & 0 & 0 & 0 & 0 & 0 & 0 & 0 & 0 & 0 \\
\hline 13.0 & 09 & 0 & 0 & 0 & 0 & 0 & 0 & 0 & 0 & 0 & 0 & 0 & 0 & 0 \\
\hline 13.5 & 08 & 0 & 0 & 0 & 0 & 0 & 0 & 0 & 0 & 0 & 0 & 0 & 0 & 0 \\
\hline 14.0 & 07 & 0 & 0 & 0 & 0 & 0 & 0 & 0 & 0 & 0 & 0 & 0 & 0 & 0 \\
\hline 14.5 & 02 & 0 & 0 & 0 & 0 & 0 & 0 & 0 & 0 & 0 & 0 & 0 & 0 & 0 \\
\hline 15.0 & 02 & 0 & 0 & 0 & 0 & 0 & 0 & 0 & 0 & 0 & 0 & 0 & 0 & 0 \\
\hline 15.5 & 0 & 0 & 0 & 0 & 0 & 0 & 0 & 0 & 0 & 0 & 0 & 0 & 0 & 0 \\
\hline 16.0 & 0 & 0 & 0 & 0 & 0 & 0 & 0 & 0 & 0 & 0 & 0 & 0 & 0 & 0 \\
\hline
\end{tabular}

Table A.1. Table of the percentage of particles accreted onto star 1 for all simulated configurations of prograde parabolic $(e=1)$ star-disc encounters. The first row contains the relative perturber masses $M_{2}^{*} / M_{1}^{*}$, the first column contains the relative periastra $r_{\mathrm{p}} / r_{\mathrm{d}}$. 